\title{
The Effects of an Anti-glare Sleeve Installed on Fluorescent Tube Lamps on Glare and Reading Comfort
}

\author{
Shiaw-Tsyr Uang ${ }^{1}$, Cheng-Li Liu ${ }^{2}$, and Mali Chang ${ }^{1}$ \\ ${ }^{1}$ Department of Industrial Engineering and Management, \\ Minghsin University of Science and Technology, Hsinchu 304, Taiwan \\ ${ }^{2}$ Department of Industrial Management, \\ Vanung University, Taoyuan 302, Taiwan \\ uang@must.edu.tw, johnny@vnu.edu.tw, mali@must.edu.tw
}

\begin{abstract}
Our previous study has demonstrated the benefits of a reflective sleeve to redirect lighting and to enhance luminous intensity of fluorescent tube lamps in certain light projecting angles. A reflective sleeve is composed of a plastic reflector and a transparent refractor. However, the intensive centralized lighting may increase the possibilities of producing glare. In this study, the transparent refractor of the sleeve is replaced with a diffuser to compose an anti-glare sleeve. This study adopts measurement, optical software simulation, and experiment methods to investigate the effects of an anti-glare sleeve on redirecting lighting and reducing glare. The results demonstrate that luminous intensity towards viewing objects of a fluorescent tube lamp enhances after adopting an anti-glare sleeve. In addition, software simulation indicates an antiglare sleeve increases light uniformity and reduces glare. The subjective evaluation also shows that florescent tube lamps with anti-glare sleeves produce less light reflection on various papers and more comfortable reading.
\end{abstract}

Keywords: Glare, Reading comfort, Fluorescent tube lamp, Lamp sleeve.

\section{Introduction}

According to previous studies, up to $20 \%$ of world's electrical energy consumption is used for lighting purposes [1]. Fluorescent lamps have the advantages of larger light emitting area, more evenly light uniformity, lower temperature on tube surface, similar color to sunlight, and longer life period than incandescent lamps do. Hence, fluorescent lamps are the most commonly and widely used artificial light sources in indoor spaces nowadays [2-4].

Our previous study has demonstrated the benefits of installing a reflective sleeve on a fluorescent tube lamp to redirect lighting and to enhance luminous intensity in certain light projecting angles around 80\% [5]. A reflective sleeve is composed of a plastic reflector and a transparent refractor to control light distribution and density. However, the intensive centralized lighting may increase the possibilities of producing glare which cause eye discomfort and/or performance decrement. 
Regarding reducing glare, Japuntich [6] found that the use of a linear polarized light source helps to minimize specular glare by darkening the reflected image of the light source on the document. Theoretical predictions and light measurement analysis of specular glare reduction were compared with empirical results from testing on a panel of humans on semi-gloss finish and matte finish papers. This study showed that with the right alteration of a polarized light source position, specular glare may be significantly reduced, and correlations exist between the theory, empirical measurements and the human response to specular glare reduction.

Osterhaus [7] reviewed and discussed the advantages and limitations of using existing glare indices for daylighting conditions. It concluded that available assessment and prediction methods are of limited practical use in daylit situations and currently have no provision for integrated systems that combine daylighting and electric lighting. This paper also presented selected findings from a case study of daylit office environments which identify a number of important design considerations.

Iwataa et al. [8] measured subjective response to intense light, or glare caused by a wide source. Three glare indices have been investigated in this study: the Building Research Station glare index, the CIE glare index and the Cornell daylight glare index. They have also examined the glare vote and have proposed a new glare evaluation scale, as well as asking the subjects to vote on the condition's acceptability. The Cornell formula most accurately predicts glare discomfort, but it is found to be inadequate for a range of wide-source glare conditions. Both the discomfort sensation and the glare ratings which they proposed correlate well with the percentage of subjects dissatisfied when looking directly at the light source.

Koga 及 Kim [9] proposed a practical method of determining the background luminance in the evaluation of discomfort glare. Two experiments were conducted, a visual sensitivity test and a glare sensitivity test. The results show that the luminance of threshold and the luminance of discomfort glare are mainly determined by the luminance of the immediate background of the source, rather than by the average background luminance.

Velds [10] tried to draw up a relation between glare assessments and measured quantities. For this purpose two test rooms were used: one room was occupied by the subject, and the required measuring equipment was placed in the other one. An electronic questionnaire was developed for these studies and installed on the computer of the subject. Continuous measurements were necessary to link subjective assessments to measured quantities that were obtained at the same time. The study showed that the vertical illumination measured near the facade and the average sky luminance measured from the back of the room are good measures to monitor visual comfort under intermediate and overcast sky conditions.

In this study, the transparent refractor of the sleeve is replaced with a diffuser to compose a newly designed lamp sleeve, which we called it an anti-glare sleeve (see Fig. 1). The reflector of this sleeve is used to redirect lighting towards viewing objects. On the other hand, the diffuser is used to diffuse lights, thus to reduce glare. Hence, the purpose of the present research is to investigate the effects of an anti-glare sleeve on redirecting lighting and reducing glare. 

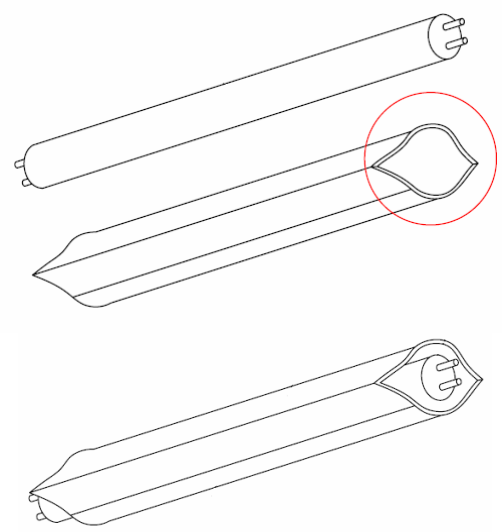

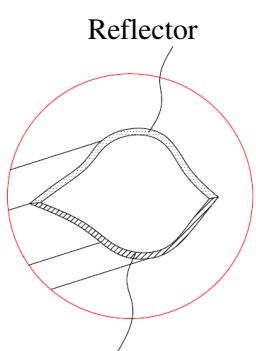

Diffuser

Fig. 1. A fluorescent tube lamp with an anti-glare sleeve

\section{Methods}

This study adopts measurement, optical software simulation, and experiment methods to investigate the effects of an anti-glare sleeve on redirecting lighting and reducing glare. These three methods are described below in this section.

First, the luminous intensity distribution curves of 10, 20 and 40 watts T8 florescent tube lamps with no sleeve, a reflective sleeve and an anti-glare sleeve in different light projecting angles $\left(0^{\circ}, 45^{\circ}, 90^{\circ}\right)$ were measured and recorded by a goniophotometer system. This measurement can obtain the maximum and average luminous intensity (unit: cd), as well as the shape of light distribution. Fig. 2 is the apparatus used in this study and their configuration.

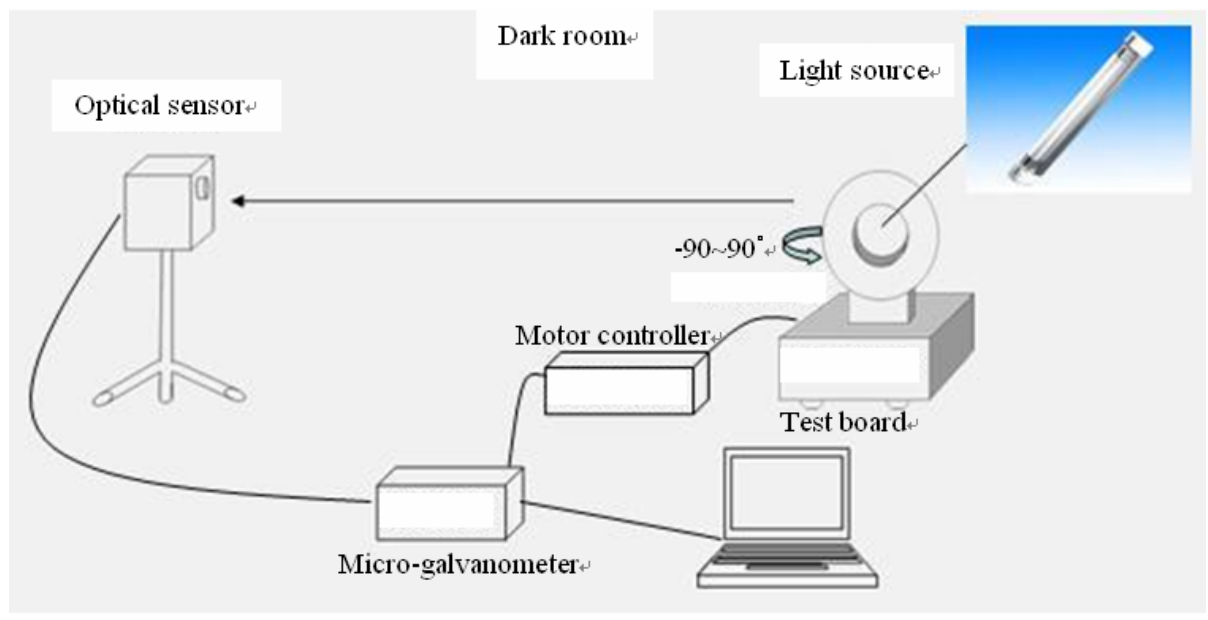

Fig. 2. The measurement apparatus and their configuration 
Next, the luminous intensity collected was imported to the optical softwareLumen Micro 2000. This software was then used to build up simulated lighting condition of one classroom and to calculate illumination levels and glare indexes in order to evaluate the effects in enhancing luminous intensity and reducing glare. Simulation has the advantage in estimating lighting conditions without installing real lamps and lighting fixtures. In addition, Lumen Micro 2000 is capable of computing the glare indexes (visual comfort probability, VCP) of the users.

Finally, 30 subjects participated in a laboratory experiment. All 30 subjects have no difficulty in discriminating colors and have normal or corrected-to-normal visual acuity. Subjects evaluated reading effects and their visual comfort while viewing words and graphs on matt finish papers, copy papers, and dowling papers with different kinds of florescent tube lamps (no sleeve, a reflective sleeve and an antiglare sleeve). Subjects experienced all nine $(3 * 3)$ experimental combinations in random order. Their evaluation was based on a 7-point Likert-type scale, which "1" denotes "strongly disagree" and "7" denotes "strongly agree". Fig. 3 is our experimental configuration.

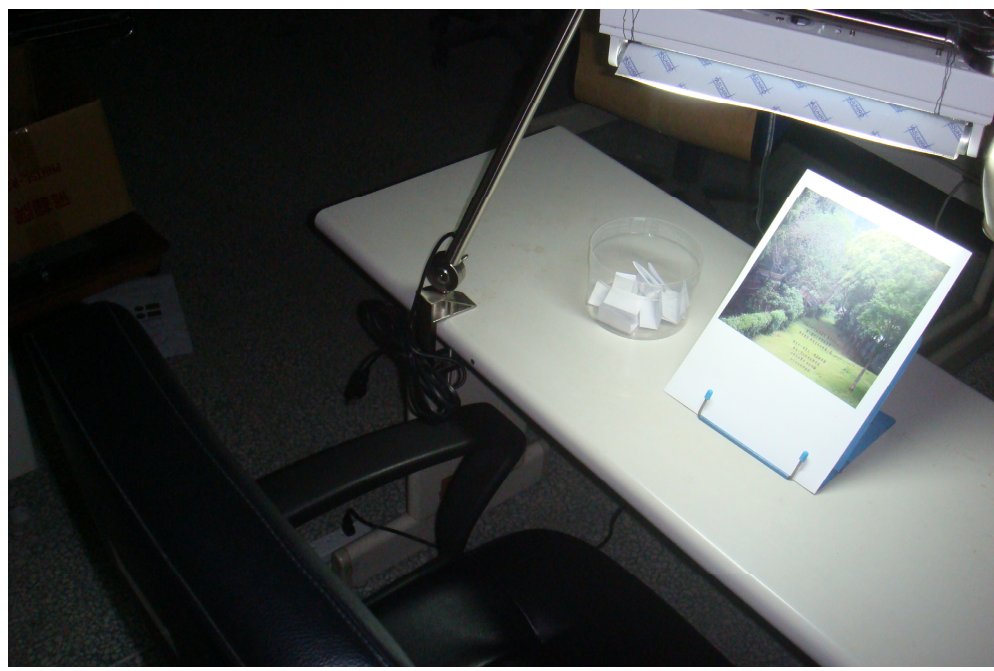

Fig. 3. The experimental configuration of this study

\section{Results}

This study adopts measurement, optical software simulation, and experiment methods to investigate the effects of an anti-glare sleeve on redirecting lighting and reducing glare. This section provides the findings from these three research methods respectively. 


\subsection{Results of Measurement}

A goniophotometer was used to collect the luminous intensity data of different watts (10W, 20W, 40W) T8 fluorescent tube lamps with different sleeve conditions (no sleeve, a reflector sleeve, an anti-glare sleeve) from $-90^{\circ}$ to $90^{\circ}$ in different light projecting angles $\left(0^{\circ}, 45^{\circ}, 90^{\circ}\right)$. 27 paired t tests were conducted to compare the difference of luminous intensity. The results indicate 25 of the $27 \mathrm{t}$ tests are statistically significant. It implies the adoption of sleeve can produce significant difference in luminous intensity.

In order to explore this finding further, the luminous intensity diagram curves from $-90^{\circ}$ to $90^{\circ}$ were draw and used to compare the shapes of lighting. These curves demonstrate similar and consistent findings; therefore, only the diagrams of a $10 \mathrm{~W}$ fluorescent tube lamp in $90^{\circ}$ with different sleeve conditions are provided in this paper (shown in Fig. 4 6). The lighting of a fluorescent tube lamp dispersed widely from $-90^{\circ}$ to $90^{\circ}$ (Fig. 4). However, with a reflective sleeve (Fig. 5) or an anti-glare sleeve (Fig. 6) cause the light distribution centralized toward the illuminated surface.

\section{Luminous Intensity Diagram Curve}

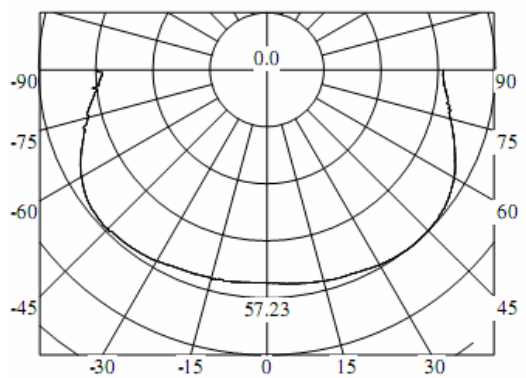

In polar coordinates

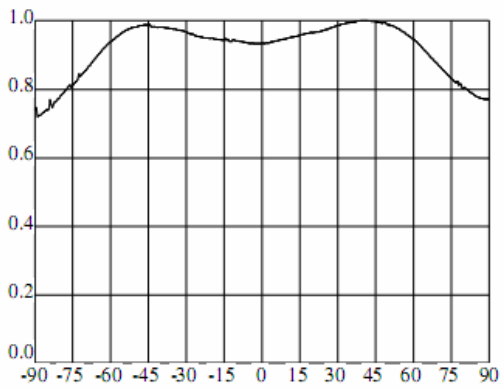

In cartesian coordinates

Fig. 4. Luminous intensity curve of a $10 \mathrm{~W}$ fluorescent tube lamp in $90^{\circ}$

\section{Luminous Intensity Diagram Curve}

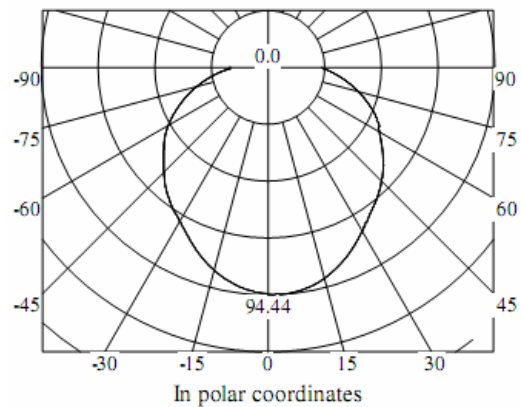

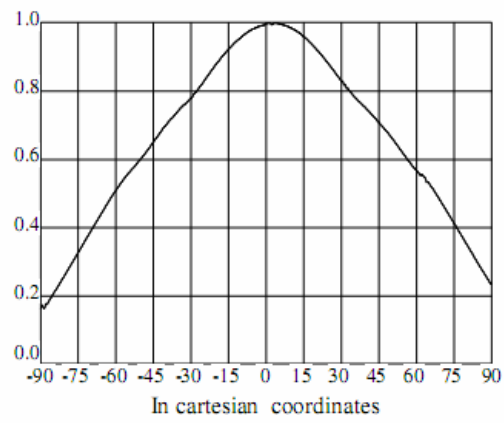

In cartesian coordinates

Fig. 5. Luminous intensity curve of a $10 \mathrm{~W}$ fluorescent tube lamp with a reflective sleeve in $90^{\circ}$ 
Luminous Intensity Diagram Curve

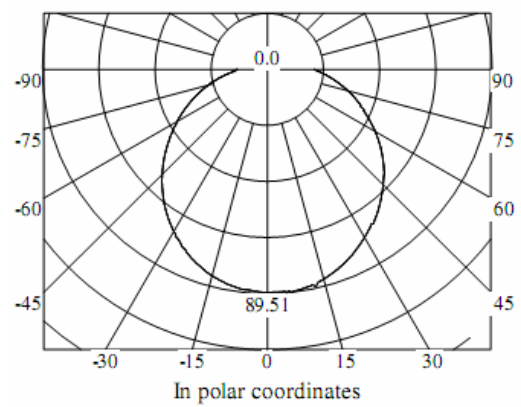

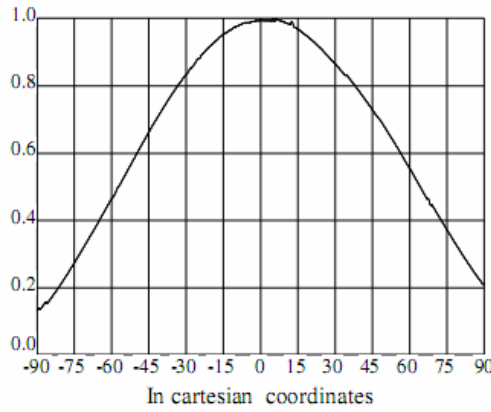

Fig. 6. Luminous intensity curve of a $10 \mathrm{~W}$ fluorescent tube lamp with an anti-glare sleeve in $90^{\circ}$

The maximum and average luminous intensity (unit: cd) in light projecting angle $0^{\circ}, 45^{\circ}$ and $90^{\circ}$ are summarized in Table 1 3. Compared to no sleeve condition, a reflective sleeve or an anti-glare sleeve increases the maximum and average luminous intensity. Besides, the increment of these two sleeves seems to have similar trends.

Table 1. The maximum and average luminous intensity in $0^{\circ}$

\begin{tabular}{|c|c|c|c|c|c|c|c|c|c|}
\hline Sleeve & \multicolumn{3}{|c|}{ No sleeve } & \multicolumn{3}{|c|}{ A reflective sleeve } & \multicolumn{3}{|c|}{ An anti-glare sleeve } \\
\hline Items & 10W & $20 W$ & 40W & $10 \mathrm{~W}$ & $20 \mathrm{~W}$ & $40 \mathrm{~W}$ & $10 \mathrm{~W}$ & $20 \mathrm{~W}$ & 40W \\
\hline $\begin{array}{l}\text { Maximum } \\
\text { cd }\end{array}$ & 55.4 & 111.8 & 179.7 & 90 & 211.5 & 373.4 & 90.1 & 219.6 & 414.9 \\
\hline $\begin{array}{l}\text { Average } \\
\text { cd }\end{array}$ & 45.6 & 91.7 & 147.8 & 74.2 & 174.3 & 307.9 & 73.7 & 180.5 & 343 \\
\hline
\end{tabular}

Table 2. The maximum and average luminous intensity in $45^{\circ}$

\begin{tabular}{|c|c|c|c|c|c|c|c|c|c|}
\hline Sleeve & \multicolumn{3}{|c|}{ No sleeve } & \multicolumn{3}{|c|}{ A reflective sleeve } & \multicolumn{3}{|c|}{ An anti-glare sleeve } \\
\hline Items & $10 \mathrm{~W}$ & $20 \mathrm{~W}$ & $40 \mathrm{~W}$ & $10 \mathrm{~W}$ & $20 \mathrm{~W}$ & $40 \mathrm{~W}$ & $10 \mathrm{~W}$ & $20 \mathrm{~W}$ & $40 \mathrm{~W}$ \\
\hline $\begin{array}{l}\text { Maximum } \\
\text { cd }\end{array}$ & 55.9 & 105.9 & 177.1 & 95.4 & 204 & 317.8 & 90.6 & 213.6 & 412.6 \\
\hline $\begin{array}{l}\text { Average } \\
\text { cd }\end{array}$ & 46.0 & 87.9 & 144.1 & 76.1 & 166.2 & 258.7 & 73.8 & 173.3 & 338.3 \\
\hline
\end{tabular}

Table 3. The maximum and average luminous intensity in $90^{\circ}$

\begin{tabular}{|l|c|c|c|c|c|c|c|c|c|}
\hline \multirow{2}{*}{$\begin{array}{l}\text { Items } \\
\text { Sleeve }\end{array}$} & \multicolumn{3}{|c|}{ No sleeve } & \multicolumn{3}{c|}{ A reflective sleeve } & \multicolumn{3}{c|}{ An anti-glare sleeve } \\
\cline { 2 - 10 } & $10 \mathrm{~W}$ & $20 \mathrm{~W}$ & $40 \mathrm{~W}$ & $10 \mathrm{~W}$ & $20 \mathrm{~W}$ & $40 \mathrm{~W}$ & $10 \mathrm{~W}$ & $20 \mathrm{~W}$ & $40 \mathrm{~W}$ \\
\hline $\begin{array}{l}\text { Maximum } \\
\text { cd }\end{array}$ & 57.2 & 121.3 & 206.7 & 94.4 & 208.5 & 347.1 & 89.5 & 215.5 & 395.7 \\
\hline $\begin{array}{l}\text { Average } \\
\text { cd }\end{array}$ & 52.9 & 111.8 & 171.1 & 74 & 159.6 & 272.4 & 73 & 174.7 & 322.8 \\
\hline
\end{tabular}




\subsection{Results of Simulation}

The simulated space of this research is a classroom of one junior high school in Taiwan. Fig. 7 is the photo of this classroom, and Fig. 8 is the same classroom build up in Lumen Micro 2000.

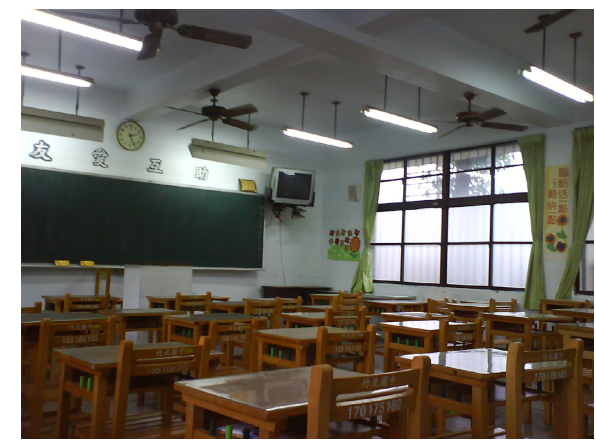

Fig. 7. The photo of the classroom being simulated

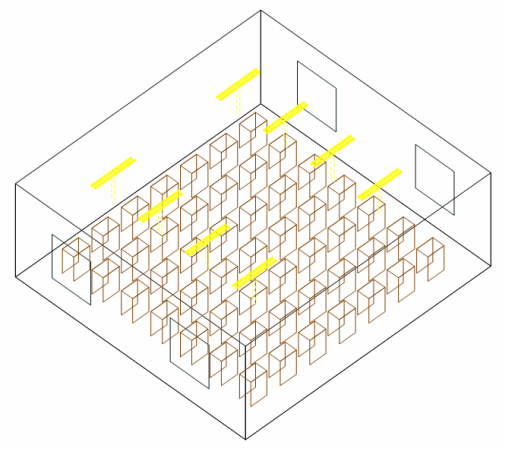

Fig. 8. The simulated classroom by Lumen Micro 2000

The average horizontal illumination measured in this classroom is 251.27 lux, which is closed to the simulated illumination, 255.7 lux. The average horizontal illumination while using reflective sleeves is estimated to be 333.9 lux by Lumen Micro 2000; and 377.2 lux while using anti-glare sleeves.

Visual comfort probability (VCP) represents the degree of visual comfort of persons. The larger the VCP value is, the smaller the glare is. Smaller max/min VCP value indicates the uniformity distribution of lighting in a space. The VCP values estimated by Lumen Micro 2000 are summarized in Table 4. An anti-glare sleeve has larger average VCP (54.7) and smaller max/min VCP (3.6). In other words, an antiglare sleeve seems to be beneficial in reducing glare.

Table 4. A summary table of the visual comfort probability (VCP) values

\begin{tabular}{|c|c|c|c|c|}
\hline Sleeve VCP & Average VCP & Maximum VCP & Minimum VCP & Max/Min VCP \\
\hline No sleeve & 50.5 & 99 & 16.4 & 6.1 \\
\hline A reflective sleeve & 50.1 & 98 & 20.1 & 4.9 \\
\hline An anti-glare sleeve & 54.7 & 97.9 & 27.1 & 3.6 \\
\hline
\end{tabular}

Unit: Percentage (\%)

\subsection{Results of Experiment}

The experiment contained two factors: three kinds of sleeve conditions (Sleeve), no sleeve, a reflective sleeve and an anti-glare sleeve; and three types of paper materials 
(Paper), matt finish papers, copy papers and dowling papers. Each participant $(\mathrm{n}=30)$ randomly experienced the sequences of all the 9 combinations of Sleeve and Paper. The $3 * 3$ within-subject two-way ANOVA analyses were conducted to examine the main effects and interactions of Sleeve and Paper on reading effects and visual comfort.

Reading effects. There are four subjective ratings related to reading effects as described in the followings.

An ANOVA on "I can clearly read the words on the paper." revealed one significant interaction between Sleeve and Paper, $\mathrm{F}_{(4,116)}=5.619, \mathrm{p}<0.01$. As shown in Fig. 9, the two-way interaction term shows that for matt finished papers, an anti-glare sleeve significantly increased the words readability (Mean=6.10) beyond the other sleeve conditions. However, the same effect was not found for copy or dowling papers.

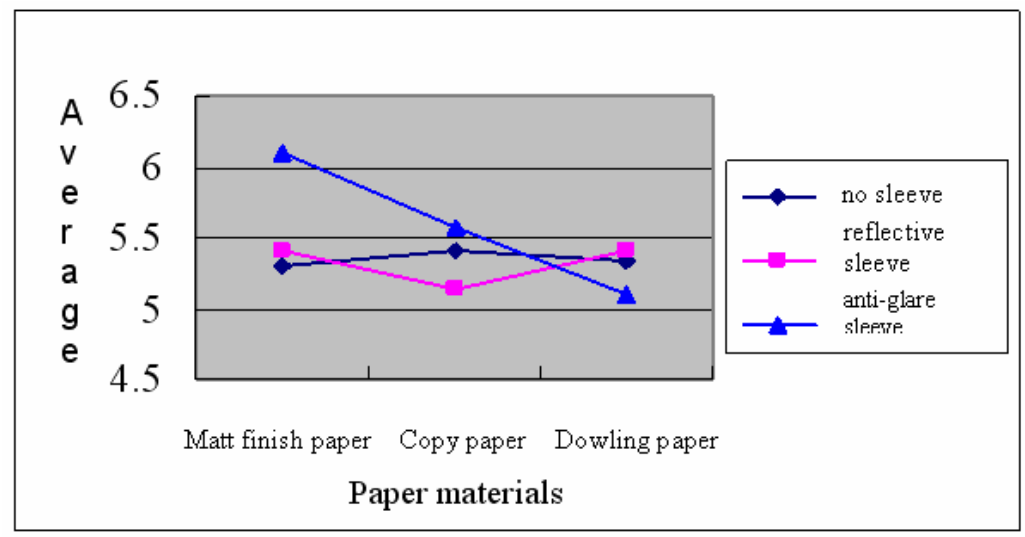

Fig. 9. The interaction between Sleeve and Paper on "I can clearly read the words on the paper."

An ANOVA on "I can easily identify the words on the paper." revealed two significant main effects: Sleeve, $\mathrm{F}_{(2,58)}=10.696, \mathrm{p}<0.01$, and Paper, $\mathrm{F}_{(2,58)}=6.990$, $\mathrm{p}<0.01$. Further analysis on Sleeve indicated an anti-glare sleeve has better words identification (Mean=5.72) than no sleeve (Mean=5.15) and a reflective sleeve $(\mathrm{Mean}=5.26)$. Further analysis on Paper indicated matt finish papers have better words identification (Mean=5.60) than copy papers (Mean=5.33) and dowling papers $($ Mean=5.20).

An ANOVA on "I feel the graph on the paper is clear." revealed one significant main effect: Sleeve, $\mathrm{F}_{(2,58)}=7.178, \mathrm{p}<0.01$. Further analysis on Sleeve indicated an anti-glare sleeve has better graph clarity (Mean=5.42) than no sleeve (Mean=4.92) and a reflective sleeve $($ Mean=4.90).

An ANOVA on "I feel the color of graph is vivid." revealed two significant main effects: Sleeve, $\mathrm{F}_{(2,58)}=21.648, \mathrm{p}<0.01$, and Paper, $\mathrm{F}_{(2,58)}=5.962, \mathrm{p}<0.01$. Further analysis on Sleeve indicated an anti-glare sleeve has better color (Mean=5.24), followed by a reflective sleeve (Mean=4.67), and no sleeve is worse (Mean=4.39). 
Further analysis on Paper indicated matt finish papers have better color (Mean=5.11) than copy papers (Mean=4.58) and dowling papers (Mean=4.61).

Visual Comfort. There are four subjective ratings related to visual comfort as described in the followings.

An ANOVA on "I feel eye fatigue." revealed two significant main effects: Sleeve, $\mathrm{F}_{(2,58)}=42.095, \mathrm{p}<0.01$, and Paper, $\mathrm{F}_{(2,58)}=5.288, \mathrm{p}<0.01$. Further analysis on Sleeve indicated no sleeve has higher eye fatigue (Mean=4.61), followed by a reflective sleeve (Mean=4.07), and an anti-glare sleeve is less eye fatigue (Mean=2.95). Further analysis on Paper indicated matt finish papers have higher eye fatigue (Mean=4.18) than copy papers (Mean=3.74) and dowling papers (Mean=3.71).

An ANOVA on "I am aware the light reflection on the paper." revealed one significant interaction between Sleeve and Paper, $F_{(4,116)}=5.751, p<0.01$, and two main effects: Sleeve, $\mathrm{F}_{(2,58)}=75.130, \mathrm{p}<0.01$, and Paper, $\mathrm{F}_{(2,58)}=60.318, \mathrm{p}<0.01$. As shown in Fig. 10, the two-way interaction term shows that an anti-glare sleeve significantly reduced light reflection regardless paper materials, and viewing matt finish papers under a reflective sleeve or no sleeve may increase the experienced light reflection.

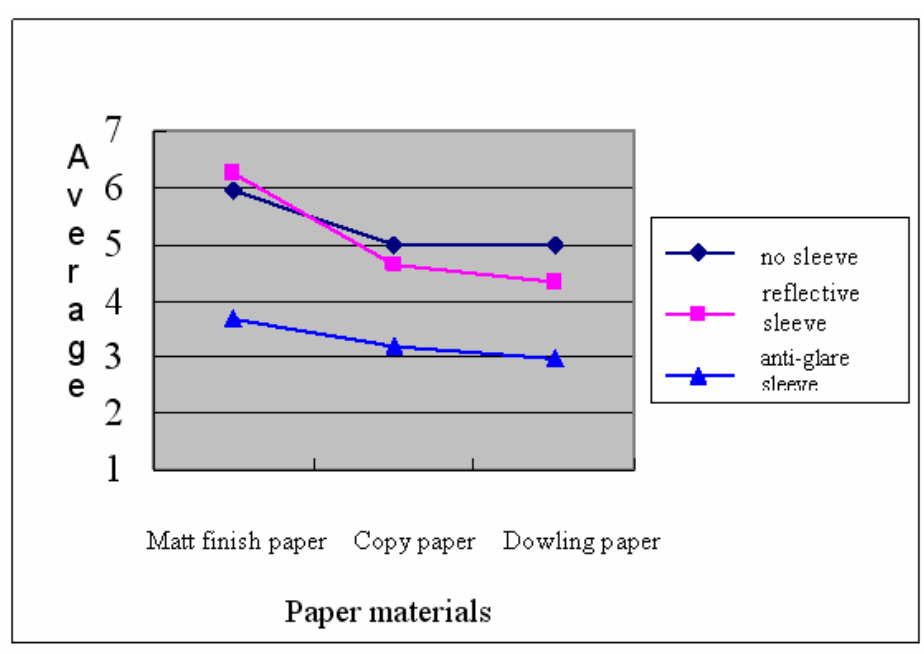

Fig. 10. The interaction between Sleeve and Paper on "I am aware the light reflection on the paper."

An ANOVA on "I feel this light source harsh to my eyes." revealed one significant main effect: Sleeve, $\mathrm{F}_{(2,58)}=67.199, \mathrm{p}<0.01$. Further analysis on Sleeve indicated no sleeve has higher harsh $($ Mean=5.20), followed by a reflective sleeve (Mean=4.04), and an anti-glare sleeve is less harsh (Mean=2.65).

An ANOVA on "I feel this lighting condition comfortable." revealed one significant main effect: Sleeve, $\mathrm{F}_{(2,58)}=75.025, \mathrm{p}<0.01$. Further analysis on Sleeve indicated no sleeve is the least comfortable (Mean=2.90), followed by a reflective sleeve $($ Mean=3.81), and an anti-glare sleeve is more comfortable (Mean=5.38). 


\section{Conclusions}

This research demonstrates a new way instead of lighting fixtures to control light distribution and glare emitted from fluorescent tube lamps by lamp sleeves. This antiglare sleeve is made of plastic material (PET), thus it is remarkable cheaper than lighting fixtures. In addition, an anti-glare sleeve is easy to install on the lamp. Especially, the findings of the present study verify the benefits of an anti-glare sleeve in increasing luminous intensity towards viewing objects, reducing glare, and generating more comfortable reading.

\section{Acknowledgments}

This research is financially supported by the National Science Council of Taiwan under contract number NSC97-2221-E-159-012.

\section{References}

1. Giris, T.E.: Some Suggestions for Photovoltaic Power Generation Using Artificial Light Illumination. Solar Energy Materials \& Solar Cells 90, 2569-2571 (2006)

2. Rea, M.S.: IESNA Lighting Handbook: Reference and Application. Illuminating Engineering Society of North America (1994)

3. Dizik, A.A.: Concise Encyclopedia of Interior Design, 2nd edn. Van Nostrand Reinhold, New York (1998)

4. Gluskin, E., Topalis, F.V., Kateri, I., Bisketzis, N.: The Instantaneous Light-intensity Function of a Fluorescent Lamp. Physics Letters A 353, 355-363 (2006)

5. Uang, S.-T., Liu, C.-C.: An Investigation of Adopting a Sleeve to Redistribute Lighting of a Fluorescent Tube Lamp. Journal of Illuminating Engineering (2009) (in Chinese)

6. Japuntich, D.A.: Polarized Task Lighting to Reduce Reflective Glare in Open-plan Office Cubicles. Applied Ergonomics 32, 485-499 (2001)

7. Osterhaus, W.K.E.: Discomfort Glare Assessment and Prevention for Daylight Applications in Office Environments. Solar Energy 79, 140-158 (2005)

8. Iwataa, T., Kimuraa, K.-I., Shukuyab, M., Takanoc, K.: Discomfort Caused by WideSource Glare. Energy and Buildings 15(3-4), 391-398 (1990-1991)

9. Kim, W., Koga, Y.: Effect of Local Background Luminance on Discomfort Glare. Building and Environment 39, 1435-1442 (2004)

10. Veldz, M.: User Acceptance Studies to Evaluate Discomfort Glare in Daylit Rooms. Solar Energy 73, 95-103 (2002) 